UB-ECM-PF-94/12

June 1994

\title{
Some Remarks on the Matching Conditions
}

\author{
D. Espriu and J. Matias \\ D.E.C.M., Facultat de Física and I.F.A.E. \\ Universitat de Barcelona \\ Diagonal, 647 \\ E-08028 Barcelona
}

\begin{abstract}
We analyze the matching conditions to determine the values that the $\mathcal{O}\left(p^{4}\right)$ coefficients of an Effective Chiral Lagrangian take in the Standard Model in the limit of a large Higgs mass, pointing out a number of subtleties that appear to have gone unnoticed previously. We apply the resulting Effective Chiral Lagrangian, including the leading two loop effects, to analyze the most recent electroweak data assuming $m_{\text {top }}=174 \pm 10 \mathrm{GeV}$.
\end{abstract}




\section{Introduction}

Using an Effective Chiral Lagrangian [1] has been a popular approach to study the Symmetry Breaking Sector of the Standard Model in recent times. The reasons for this popularity are twofold. On the one hand, some calculations concerning the scattering of longitudinally polarized $W$ 's and $Z$ 's are greatly simplified in the limit where the Higgs mass is large if, instead of the full Standard Model, one just replaces these longitudinal degrees of freedom by the corresponding Goldstone bosons[2] and works with the self-interaction part of the scalar sector of the Standard Model. In the limit where the Higgs is very massive this self-interaction sector becomes a non-linear sigma model. Secondly, it was realized some time ago[3,4] that if one assumes a certain gap between the electroweak scale $G_{F}^{-1}$ and new resonances either scalar, such as the Higgs (in the minimal Standard Model), or vector-like, such as e.g. technirhos (in composite models), it was possible to parametrize in full generality the Symmetry Breaking Sector by the $\mathcal{O}\left(p^{4}\right)$ coefficients of an Effective Chiral Lagrangian describing the interactions of the Goldstone bosons associated to the breaking of the group $S U(2)_{L} \times S U(2)_{R}$ down to its diagonal subgroup.

We have examined the way these $\mathcal{O}\left(p^{4}\right)$ coefficients are determined in the Standard Model and we have found a number of difficulties in the way the problem is usually addressed. These can be summarized as follows: a) at what level one should impose matching between the effective and fundamental theory (S-matrix elements, light particle 1PI Green functions, connected Green functions)? b) should one also consider gauge non-invariant effective operators in the effective theory since gauge invariance is lost in Green functions once the gauge is fixed?

The experimental precision for some experiments sensitive to these coefficients is such [5] that the days of order-of-magnitude estimates are gone. It is essential to be able to determine as precisely as possible the values of these $\mathcal{O}\left(p^{4}\right)$ coefficients in the different models one chooses to compare with the experimental data. The differences are often minute, but this is where the clues as to what lies beyond the Standard Model are.

We have used the most recent available electroweak data, combined with the preliminary determination of the top quark mass by CDF, to set bounds on the values of the Effective Chiral Lagrangian. The 'oblique' corrections - by far the dominant ones - turn out to be sensitive in a non-ambiguous manner to only one combination of coefficients of this effective theory. Our analysis, including the leading two loop effects, is presented in the last section.

\section{Changing Variables in the Standard Model}

In the usual representation of the Symmetry Breaking Sector of the Standard Model, the Goldstone bosons transform linearly under the $S U(2)_{L} \times U(1)_{Y}$ gauge group. However, if one is interested in a comparison with an effective lagrangian approach where the Goldstone bosons transform non-linearly it seems natural to implement a change of variables in the Standard Model itself to make the goldstones transform non-linearly too. In this way the identification of fields and Green functions will be clearer.

We write the Weinberg-Salam model (without fermions) in the following form

$$
\mathcal{L}_{S M}=-\frac{1}{2} \operatorname{Tr} W_{\mu \nu} W^{\mu \nu}-\frac{1}{4} B_{\mu \nu} B^{\mu \nu}+\frac{1}{4} \operatorname{Tr} D_{\mu} M^{\dagger} D^{\mu} M-\frac{1}{4} \lambda\left(\frac{1}{2} \operatorname{Tr} M^{\dagger} M+\frac{\mu^{2}}{\lambda}\right)^{2}
$$


$M$ collects the scalar fields. Writing $M=\sigma+i \vec{\tau} \vec{\omega}$ one recovers the familiar lagrangian. Under the local $S U(2)_{L} \times U(1)_{Y}$ group $M$ transforms as

$$
M^{\prime}(x)=e^{i \frac{\vec{\alpha} \vec{\tau}}{2}} M(x) e^{-i \frac{\beta \tau^{3}}{2}}
$$

The covariant derivative $D_{\mu}$ acts on $M$ as

$$
D_{\mu} M=\partial_{\mu} M+i g W_{\mu} M(x)-i g^{\prime} B_{\mu} M(x) \frac{\tau^{3}}{2}
$$

$B_{\mu}$ and $W_{\mu}=\frac{1}{2} W_{\mu}^{i} \tau^{i}$ are the vector boson fields, and $B_{\mu \nu}$ and $W^{\mu \nu}$ are the corresponding field strength tensors. The fields $W_{\mu}^{3}$ and $B_{\mu}$ are a combination of the physical degrees of freedom $A_{\mu}, Z_{\mu}$

$$
A_{\mu}=W_{\mu}^{3} s_{w}+B_{\mu} c_{w} \quad Z_{\mu}=W_{\mu}^{3} c_{w}-B_{\mu} s_{w}
$$

$c_{w}$ and $s_{w}$ being the cosinus and sinus of the Weinberg angle, respectively. In the on-shell scheme, which we shall use, $c_{w} \equiv M_{W} / M_{Z}$.

One should add to (2.1) the gauge-fixing and Faddeev-Popov terms

$$
\begin{aligned}
\mathcal{L}_{G F} & =-\frac{1}{2 \xi_{W}} \sum_{i=1,3}\left(\partial^{\mu} W_{\mu}^{i}+\frac{i}{4} g v \xi_{W} \operatorname{Tr} \tau^{i} M\right)^{2}-\frac{1}{2 \xi_{B}}\left(\partial^{\mu} B_{\mu}-\frac{i}{4} g^{\prime} v \xi_{B} \operatorname{Tr} \tau^{3} M\right)^{2} \\
\mathcal{L}_{F P}= & \partial^{\mu} c_{0}^{\dagger} \partial_{\mu} c_{0}+\partial^{\mu} c_{i}^{\dagger} \partial_{\mu} c_{i}-\frac{1}{8} g^{\prime 2} v \xi_{B} c_{0}^{\dagger} \operatorname{Tr} M c_{0}+g c_{i}^{\dagger}\left(\partial^{\mu} W_{\mu}^{k} \epsilon^{i k j}-\frac{1}{8} g v \xi_{W} \operatorname{Tr} \tau^{i} \tau^{j} M\right) c_{j} \\
& +\frac{1}{8} \sqrt{g g^{\prime}} v\left(g^{\prime} \xi_{B} c_{0}^{\dagger} c_{i}+g \xi_{W} c_{i}^{\dagger} c_{0}\right) \operatorname{Tr} \tau^{3} \tau^{i} M
\end{aligned}
$$

In Landau gauge $(\xi=0)$ ghosts decouple from Goldstone bosons.

We are free to choose a different parametrization for the matrix $M$, implying a field redefinition. Not any transformation leaves the S-matrix elements between physical states unchanged, however. In the functional integral $\mathcal{Z}=\int \mathcal{D} \phi e^{i \mathcal{S}+J \phi}$, where $\phi$ and $J$ collectively stand for field and sources, respectively, such a field redefinition will induce a jacobian. It was demonstrated in [6] that if this jacobian is the identity when all fields are set to zero then the corresponding transformation is an allowed one. One of such allowed field redefinitions in the Standard Model is precisely the one mapping the linear onto the non-linear realization

$$
M=(v+\rho) U \quad U=\exp i \frac{\vec{\pi} \vec{\tau}}{v}
$$

where we already allow for a non-zero expectation value of the matrix $M$ by introducing $v$. In the Standard Model, $v=\sqrt{-\mu^{2} / \lambda} \simeq 250 \mathrm{GeV}$.

We have

$$
\begin{aligned}
\sigma & =(\rho+v) \cos \frac{\pi}{v}-v=\rho-\frac{1}{2 v} \pi^{2}-\frac{\rho}{2 v^{2}} \pi^{2}+\ldots \\
\omega^{i} & =(\rho+v) \frac{\pi^{i}}{\pi} \sin \frac{\pi}{v}=\pi^{i}+\frac{\rho}{v} \pi^{i}-\frac{1}{6 v^{2}} \pi^{2} \pi^{i}+\ldots
\end{aligned}
$$


with $\pi=\sqrt{\sum_{i=1,3}}\left(\pi^{i}\right)^{2}$. The jacobian of the change is

$$
J\left(\rho, \pi^{i}\right)=\left|\frac{\partial\left(\sigma, \omega^{i}\right)}{\partial\left(\rho, \pi^{j}\right)}\right|=\frac{1}{v}(\rho+v)^{3} \frac{\sin ^{2} \frac{\pi}{v}}{\pi^{2}}
$$

so indeed $J(0,0)=1$, checking that the transformation is an allowed one.

In fact, in the non-linear realization one expects the covariant group measure $d \mu(U)=$ $\prod d \pi^{i} \sqrt{\operatorname{det} g}$ to appear. Here $g$ is the group metric, given by

$$
g_{j k}=\frac{\delta^{2}}{\delta\left(\partial_{\nu} \pi^{j}\right) \delta\left(\partial^{\nu} \pi^{k}\right)}\left(\frac{v^{2}}{4} \operatorname{Tr}_{\mu} U^{\dagger} \partial^{\mu} U\right)=v^{2} \frac{\sin ^{2} \frac{\pi}{v}}{\pi^{2}}\left(\delta_{j k}-\frac{\pi_{j} \pi_{k}}{\pi^{2}}\right)+\frac{\pi_{j} \pi_{k}}{\pi^{2}}
$$

Working out $\sqrt{\operatorname{det} g}$ one finds

$$
\sqrt{\operatorname{det} g}=v^{2} \frac{\sin ^{2} \frac{\pi}{v}}{\pi^{2}}
$$

which is indeed contained in (2.10). The jacobian (2.10) can be exponentiated

$$
\operatorname{det} J=\exp \delta^{(4)}(0) \operatorname{Tr} \ln J
$$

In dimensional regularization $\delta^{4}(0)$ is zero. But in other possible regularization methods[7] this term will generate some tadpoles that will be needed to yield a consistent result[8]. We will only use dimensional regularization here and accordingly we will ignore the jacobian altogether.

After substituting the parametrization (2.7) in (2.1) one finds

$$
\begin{aligned}
\mathcal{L}_{S M}= & \frac{1}{2} \partial_{\mu} \rho \partial^{\mu} \rho-\rho \lambda v\left(v^{2}+\frac{\mu^{2}}{\lambda}\right)-\frac{1}{2} \rho^{2}\left(\mu^{2}+3 v^{2} \lambda\right)-\lambda v \rho^{3}-\frac{1}{4} \lambda \rho^{4} \\
& +\frac{1}{4}(\rho+v)^{2} \operatorname{Tr} D_{\mu} U^{\dagger} D^{\mu} U+\mathcal{L}_{G F}^{\prime}+\mathcal{L}_{F P}^{\prime}
\end{aligned}
$$

The primes in $\mathcal{L}_{F P}$ and $\mathcal{L}_{G F}$ indicate that they also change under the redefinition (2.7).

The couplings involving Goldstone bosons, collected in the matrix $U$, have changed completely when compared to the linear realization. All these couplings now involve derivatives and there are an infinite number of them since we have a non-linear theory. Yet, this non-linear theory is strictly equivalent to the Standard Model. The couplings involving at least one gauge field remain unchanged up to three fields, but for four fields and beyond this is no longer the case. For instance, there is no vertex $W^{\mu+} W_{\mu}^{-} \pi^{+} \pi^{-}$. Some new vertices appear, e.g. $\partial_{\mu} \pi^{3} Z^{\mu} \rho^{2}$, and others change their coefficients. The vertex that in the old variables was $\frac{i}{2} g g^{\prime} s_{w} Z_{\mu} W_{\mu}^{+} \omega^{-} \sigma$ now gets an extra factor of 2 and becomes $i g g^{\prime} s_{w} Z_{\mu} W_{\mu}^{+} \pi^{-} \rho$. As befits a non-linear theory, we have vertices with five, six, etc. fields, but they do not contribute to the Green functions we will be interested in at the one loop level. Of course, the different coefficients have just the right values so as to render a renormalizable and unitary theory as the Standard Model should be. 


\section{Heavy Higgs Limit}

The parametrization (2.7) is particularly useful in discussing the limit, within the minimal Standard Model, when the Higgs particle is very heavy. All the $M_{H}$ (or $\lambda$ ) dependence is contained only in the propagator and self-interactions of the $\rho$ field, while in the linear realization there are $\lambda$ dependences in any scalar vertex.

The $\rho$ field itself interacts with the Goldstone bosons and the gauge bosons through the operator $O_{1}(x)=\operatorname{Tr} D_{\mu} U^{\dagger} D^{\mu} U$ and through the gauge-fixing and Fadeev-Popov terms. The familiar 't Hooft gauge-fixing term (2.5) in the non-linear variables reads

$\mathcal{L}_{G F}=-\frac{1}{2 \xi_{W}} \sum_{i=1,3}\left(\partial^{\mu} W_{\mu}^{i}+\frac{i}{4} g v \xi_{W}(v+\rho) \operatorname{Tr} \tau^{i} U\right)^{2}-\frac{1}{2 \xi_{B}}\left(\partial^{\mu} B_{\mu}-\frac{i}{4} g^{\prime} v \xi_{B}(v+\rho) \operatorname{Tr} \tau^{3} U\right)^{2}$

Unlike the usual formulation the Higgs field $\rho$ appears in the gauge-fixing term. Of course one could well have chosen some other gauge-fixing term, since amplitudes are after all gauge independent, but, in practice, we will be interested in making comparisons at other levels. Most calculations in the Standard Model are done in the 't Hooft gauge. In fact, the comparison between theory and experiment is usually done for LEP physics in the 't Hooft-Feynman gauge $(\xi=1)[9,10]$. 'Observables' such as the effective Weinberg angle $\bar{s}_{w}^{2}$ are actually gauge dependent[13]. The $\rho$ couplings are simplest in the 't Hooft-Landau gauge where the Faddeev-Popov term does not lead to new Higgs interactions and the additional $\rho$ dependence appears only through the coupling

$$
\rho(x) O_{2}(x)=-\frac{i v}{4} \rho\left(g \sum_{i=1,3} \partial^{\mu} W_{\mu}^{i} \operatorname{Tr} \tau^{i} U-g^{\prime} \partial^{\mu} B_{\mu} \operatorname{Tr} \tau^{3} U\right)
$$

One can now formally perform the functional integration over the Higgs field. The result of such an integration will be a non-local effective lagrangian of the type

$$
\int d k_{1} \int d k_{2} \ldots \int d k_{n} G_{\lambda}\left(k_{1}, k_{2}, \ldots, k_{n}\right) \hat{O}\left(k_{1}\right) \hat{O}\left(k_{2}\right) \ldots \hat{O}\left(k_{n}\right)
$$

where $G_{\lambda}\left(k_{1}, k_{2}, \ldots k_{n}\right)$ are Green functions that can be computed in a scalar field theory involving only the $\rho$ field without any reference to the gauge fields or Goldstone bosons. These Green functions will depend on $\lambda$ (or $M_{H}$ ) and an obviously important question is which is the behaviour of $G_{\lambda}\left(k_{1}, k_{2}, \ldots, k_{n}\right)$ when $\lambda \rightarrow \infty$. On general grounds we expect (3.3) to become a local action in that limit.

A delicate point is whether one is allowed to take the $\lambda \rightarrow \infty$ limit directly in the nonlocal lagrangian (3.3) and use the resulting local effective field theory to compute quantum fluctuations for the remaining fields[14]. Doing so would require the uniform convergence of any momentum integral in which (3.3) is inserted, a strong requirement that it is not always fulfilled. After introducing the usual counterterms (e.g. using the on-shell scheme[912] in the Standard Model) one is able to make all integrals convergent, but typically they will be only conditionally convergent, being the difference of two logarithmically divergent integrals. 
In the on-shell scheme, if one considers observables that depend only on renormalized self-energies (like the celebrated 'oblique' corrections [9] contained in $\Delta r, \Delta \kappa$ and $\Delta \rho$ )

$$
\hat{\Sigma}\left(k^{2}\right)=\Sigma\left(k^{2}\right)-\Sigma\left(M^{2}\right)+\delta Z_{2}\left(k^{2}-M^{2}\right)
$$

only one integral that depends on $M_{H}$ (actually $\sim \log M_{H}$ ) and that is not uniformly convergent appears. Thus combinations that are finite in the large $M_{H}$ limit are combinations where the potentially dangerous integral actually drops. In these combinations (which, by the way, are the ones unambiguosly predictable by an Effective Chiral Lagrangian[4,7]) we are free to take the $M_{H} \rightarrow \infty$ limit before integrating over the light degrees of freedom, simplifying the calculation considerably. This was the method used in [15] to determine the contribution of the Standard Model to some LEP observables in the large $M_{H}$ limit.

Unfortunately, it is not justified to retain only the leading terms in the $1 / M_{H}$ expansion of (3.3) for the three and four point functions. In the on-shell scheme the renormalized three and four point functions and their related observables will only be conditionally convergent, in general. We have to keep the full non-local effective action (3.3). (This point was overlooked in [15], but subsequently realized in [16].) To determine the coefficients of the effective theory reproducing the Standard Model we will use the matching conditions, which will be discussed in the next section.

It may be argued that one could expand the non-local action in inverse powers of $M_{H}$ anyway provided that a physical scale $\Lambda$ is introduced as cut-off. This is certainly correct, since all integrals are then finite and well behaved. Then one obtains a local action, equivalent to (3.3) up to scales $k^{2} \sim \Lambda^{2}$ with some definite values of the coefficients in this effective action. (These coefficients, by the way, need not coincide with those obtained by the use of the matching conditions, since the latter contain some contribution from the light particles.) The above procedure, however, for a gauge theory is very difficult to implement in an invariant way and we shall not pursue this approach further here. Dimensional regularization is the most useful regulator for gauge theories and in dimensional regularization there is no manifest decoupling of the heavy modes.

\section{Matching Conditions}

We want to construct an effective theory that reproduces the results of the Standard Model without the Higgs. An obvious requirement this effective theory should meet is to reproduce the same S-matrix elements. This is certainly a necessary condition, but it is not the most useful way to proceed. For instance, if we want to compare the fundamental and effective theories at some intermediate steps (e.g. at the level of the 'oblique' corrections) we shall need to deal with Green functions defined off-shell both in the fundamental and in the effective theory. Furthermore, to express these 'observables' in terms of the same set of parameters $\left(\alpha, M_{W}, M_{Z}\right)$ it will be mandatory to renormalize both theories with the same conventions, requiring again off-shell Green functions.

It is often stated[17] that the matching can be done at the level of the quantum effective action for the light fields; that is, at the level of the (light-fields) irreducible Green functions. This is not quite correct. Let us see why. 
From the generating functional $W=\log Z$ the renormalized connected Green functions of the theory are obtained

$$
G_{c}\left(x_{1}, x_{2}, \ldots, x_{n} ; \mu\right)=\left.\frac{\delta^{n} W}{\delta J\left(x_{1}\right) \delta J\left(x_{2}\right) \ldots \delta J\left(x_{n}\right)}\right|_{J=0}
$$

$\mu$ is some renormalization scale, chosen to be below the mass of the particle we wish to integrate out, in our case $\mu<M_{H}$. Since the gauge fields have not been modified by the change of variables (2.7), the connected Green functions with only external gauge fields should coincide when evaluated in the variables $(\sigma, \omega)$ or $(\rho, \pi)$.

This is not the case if one considers only one-particle irreducible Green functions. The Legendre transform involves the scalar fields too and these have changed. For instance, the 1PI Green functions $\Gamma_{Z W W}$ and $\Gamma_{A W W}$ computed in the $(\sigma, \omega)$ or $(\rho, \pi)$ variables at one loop change. In the large $M_{H}$ limit, the differences between the linear $(\sigma, \omega)$ and non-linear $(\rho, \pi)$ realization, denoted by $L$ and $N L$, respectively, are at one loop

$$
\begin{gathered}
\Gamma_{Z W W}^{N L}-\Gamma_{Z W W}^{L}=c_{w} \frac{1}{8} \frac{g g^{2}}{16 \pi^{2}}\left(p_{2 \mu} g_{\lambda \nu}-p_{3 \nu} g_{\lambda \mu}\right) \\
\Gamma_{A W W}^{N L}-\Gamma_{A W W}^{L}=-s_{w} \frac{1}{8} \frac{g^{3}}{16 \pi^{2}}\left(p_{2 \mu} g_{\lambda \nu}-p_{3 \nu} g_{\lambda \mu}\right)
\end{gathered}
$$

The culprit is one of the vertices containing four fields that have changed in the non-linear realization. (The full $M_{H}$ dependence of these 1PI Green functions at one loop in the linear realization can be found in [10].) This simple example should suffice to convince us that matching the Standard Model to an effective theory by demanding the equality of the 1PI Green functions is not correct, since a mere change of variables (that does not affect the S-matrix elements) in the Standard Model itself already changes these Green functions.

Of course when we put everything together we must recover the same connected functions with external gauge fields. One can check this point easily for the connected Green function $\left\langle Z W^{+} W^{-}\right\rangle$and $\left\langle A W^{+} W^{-}\right\rangle$. To find the connected Green functions one should add to Fig.1a the reducible diagrams of Fig.1b. It turns out that the 1PI Green function $\Sigma_{W \pi}$ also changes when we go to the $(\rho, \pi)$ variables; there appears a new piece proportional to the squared momentum of the internal $\pi$ field that cancels the $1 / p_{2}^{2}$ of the propagator, yielding a local contribution making up for the differences (4.2) and (4.3).

The matching conditions between an Effective Chiral Lagrangian (ECL) and the Symmetry Breaking sector of the Standard Model (SM) have therefore to be imposed at the level of renormalized connected Green functions for external gauge fields (or on arbitrary Smatrix elements between physical states, of course). We will therefore tentatively demand that

$$
G_{\mu_{1}, \mu_{2}, \ldots, \mu_{n}}^{S M}\left(x_{1}, x_{2}, \ldots, x_{n} ; \mu\right)=G_{\mu_{1}, \mu_{2}, \ldots, \mu_{n}}^{E C L}\left(x_{1}, x_{2}, \ldots, x_{n} ; \mu\right)
$$

\section{Gauge Invariance and Matching Conditions}

The effective low energy theory that one gets after integrating out the heavy degrees of freedom in the Standard Model, or, for that matter, in any theory with the same local 
symmetry and the same $S U(2)_{L} \times S U(2)_{R} \rightarrow S U(2)_{V}$ breaking pattern, is the gauged non-linear sigma model

$$
\mathcal{L}^{e f f}=-\frac{1}{2} \operatorname{Tr} W_{\mu \nu} W^{\mu \nu}-\frac{1}{4} B_{\mu \nu} B^{\mu \nu}+\frac{v^{2}}{4} \operatorname{Tr} D_{\mu} U^{\dagger} D^{\mu} U+\sum_{i=0,13} a_{i} \mathcal{L}_{i}+\mathcal{L}_{G F}+\mathcal{L}_{F P}
$$

A complete set of the operators $\mathcal{L}_{i}$ up to four derivatives was given in [1]. Some of the operators are custodially invariant, like those corresponding to the coefficients $a_{1}-a_{5}$ (in the notation of $[18,19]$ which we follow), while others, such as $a_{0}$ and $a_{6}-a_{13}$, are not. All of them are gauge invariant operators.

This is a non-renormalizable theory but it may be rendered finite at $\mathcal{O}\left(p^{4}\right)$ by redefining the $a_{i}$ coefficients (only a few of them pick up divergent counterterms actually[1]). The value (at scale $\mu$ ) of these coefficients is fixed by demanding the agreement with the Standard Model, thus trading the dependence in the scale $\mu$ by $M_{H}$. In the Standard Model therefore the bare coefficients are of the form

$$
a_{i}=\frac{1}{16 \pi^{2}}\left(c_{i}^{1}\left(C_{\epsilon}-\log \frac{M_{H}^{2}}{\mu^{2}}\right)+c_{i}^{2}\right)
$$

with $C_{\epsilon}=\frac{2}{\epsilon}-\gamma+\ln 4 \pi$. In another theory, $M_{H}^{2}$ is replaced by some other scale $\Lambda$.

It is obvious that, in the Standard Model after gauge-fixing the action, gauge noninvariant terms are introduced. In fact, the l.h.s. of the matching conditions (4.4) is gauge dependent. Gauge non-invariant pieces are also generated on the r.h.s. since in the effective theory one needs to impose some gauge-fixing condition as well. One might use gauge conditions such as e.g.

$$
\begin{gathered}
\mathcal{L}_{G F}^{1}=-\frac{1}{2 \xi_{W}} \sum_{i=1,3}\left(\partial^{\mu} W_{\mu}^{i}+\frac{i}{4} g v^{2} \xi_{W} \operatorname{Tr} \tau^{i} U\right)^{2}+\ldots \\
\mathcal{L}_{G F}^{2}=-\frac{1}{2 \xi_{W}} \sum_{i=1,3}\left(\partial^{\mu} W_{\mu}^{i}-\frac{1}{2} g v \xi_{W} \pi^{i}\right)^{2}+\ldots
\end{gathered}
$$

In fact, at the one loop level, for the Green functions we are interested both are equivalent. At this point, it is not obvious at all that the gauge non-invariant pieces that are generated at one loop in the Standard Model with the usual 't Hooft-type gauge should be the same that appear from a one-loop calculation with the pieces of $\mathcal{O}\left(p^{2}\right)$ in $\mathcal{L}^{\text {eff }}$ using one of the gauge-fixings (5.3) or (5.4). Rather, one will have to 'fine tune' the gauge-fixing in the effective theory to accomplish that. In other words, one should also include at $\mathcal{O}\left(p^{4}\right)$ some gauge non-invariant operators on the r.h.s of the matching conditions. If not, the matching conditions will overdetermine the coefficients in the Effective Chiral Lagrangian and lead to inconsistencies.

This is an unwelcome complication. Either we keep all BRS-invariant operators on the r.h.s of the matching conditions or we eliminate from the Green functions to be matched the gauge dependent structures. Clearly the latter is the simplest one and the one we take. 
We shall therefore project the connected Green functions on both sides of (4.4) on their tranversal components, by multiplying them with the factor

$$
\prod_{i=1}^{n}\left(g^{\nu_{i} \mu_{i}}-\frac{k_{i}^{\nu_{i}} k_{i}^{\mu_{i}}}{k_{i}^{2}}\right)
$$

This automatically eliminates all gauge-non invariant terms. Of course even transverse parts may depend on the value of the gauge parameter $\xi$. Fortunately, it can be easily seen[19] that at the one loop level in the limit of a large Higgs mass[12] all dependence on $\xi$ drops in diagrams containing at least one Higgs internal line. In the $M_{H} \rightarrow \infty$ limit the transverse projection of the set of diagrams in the Standard Model that contains the Higgs field; i.e. the set of diagrams whose contribution will be implemented by the coefficients $a_{i}$ forms a gauge invariant subset. One is then free to determine these coefficients using, for instance, $\xi=0$ where the chiral properties of the effective theory are manifest $[1,18]$. (The gauge-fixing term (5.3) respects the global $S U(2)_{L} \times S U(2)_{R}$ invariance in this gauge.)

Let us now write explicitly the matching conditions for the two point functions. The renormalization constants for the fields and coupling constants are defined in the usual way following the on-shell prescriptions on both sides of (4.4). The relevant diagrams will be those not common to both sides of the equation and surviving the large Higgs mass limit. They are discussed in [19]. We do not consider tadpole diagrams since they are exactly cancelled by redefining the second term in (2.14).

$$
\begin{aligned}
\Delta \hat{\Sigma}_{W W}=- & \frac{g^{2} v^{2}}{4}\left(\Delta Z_{\pi}-2 \frac{\Delta g}{g}-2 \frac{\Delta v}{v}\right)-\frac{g^{2}}{16 \pi^{2}}\left(\frac{1}{8} M_{H}^{2}-g^{2} v^{2} \frac{3}{16}\left(C_{\epsilon}-\log \frac{M_{H}^{2}}{\mu^{2}}+\frac{5}{6}\right)\right) \\
& +q^{2}\left(\Delta Z_{W}+\frac{g^{2}}{16 \pi^{2}} \frac{1}{12}\left(C_{\epsilon}-\log \frac{M_{H}^{2}}{\mu^{2}}+\frac{5}{6}\right)\right)=0 \\
\Delta \hat{\Sigma}_{\gamma \gamma}= & q^{2}\left(s_{w}^{2} \Delta Z_{W}+c_{w}^{2} \Delta Z_{B}-s_{w}^{2} g^{2}\left(a_{8}-2 a_{1}\right)\right)=0 \\
\Delta \hat{\Sigma}_{Z Z}=- & \frac{g^{2} v^{2}}{4 c_{w}^{2}}\left(\Delta Z_{\pi}-2 c_{w}^{2} \frac{\Delta g}{g}-2 s_{w}^{2} \frac{\Delta g^{\prime}}{g^{\prime}}-2 \frac{\Delta v}{v}+2 a_{0}\right) \\
& -\frac{g^{2}}{16 \pi^{2} c_{w}^{2}}\left(\frac{1}{8} M_{H}^{2}-\frac{g^{2} v^{2}}{c_{w}^{2}} \frac{3}{16}\left(C_{\epsilon}-\log \frac{M_{H}^{2}}{\mu^{2}}+\frac{5}{6}\right)\right) \\
& +q^{2}\left(c_{w}^{2} \Delta Z_{W}+s_{w}^{2} \Delta Z_{B}-c_{w}^{2} g^{2} a_{8}-2 s_{w}^{2} g^{2} a_{1}-\left(g^{2}+g^{2}\right) a_{13}\right. \\
& \left.+\frac{g^{2}}{16 \pi^{2} c_{w}^{2}} \frac{1}{12}\left(C_{\epsilon}-\log \frac{M_{H}^{2}}{\mu^{2}}+\frac{5}{6}\right)\right)=0 \\
\hat{\Sigma}_{\gamma Z}=-g g^{\prime} & \frac{v^{2}}{4}\left(\frac{\Delta g^{\prime}}{g^{\prime}}-\frac{\Delta g}{g}\right)+q^{2} s_{w} c_{w}\left(\Delta Z_{W}-\Delta Z_{B}-g^{2} a_{8}\right)+q^{2}\left(c_{w}^{2}-s_{w}^{2}\right) g g^{\prime} a_{1}=0
\end{aligned}
$$

where $\Delta$ means the difference between any quantity (self-energy, renormalization constant) evaluated in the Standard Model minus the same quantity evaluated in $\mathcal{L}^{e f f}$. Since we 
work in the on-shell scheme and the renormalization constants of both theories have been generated by the same renormalization conditions ${ }^{1}$ we know [9] that they can be expressed in terms of the unrenormalized self-energies. One can calculate their difference in both theories

$$
\begin{gathered}
\Delta Z_{W}=\frac{1}{s_{w}^{2}}\left(s_{w}^{4}-c_{w}^{4}\right) g^{2}\left(a_{8}+a_{13}\right)-2 g^{2}\left(a_{1}+a_{13}\right)-2 \frac{c_{w}^{2}}{s_{w}^{2}} a_{0}+\frac{g^{2}}{16 \pi^{2}} \frac{5}{6}\left(C_{\epsilon}-\log \frac{M_{H}^{2}}{\mu^{2}}+\frac{5}{6}\right) \\
\Delta Z_{B}=g^{2}\left(a_{8}+a_{13}\right)+2 a_{0}-\frac{g^{\prime 2}}{16 \pi^{2}} \frac{5}{6}\left(C_{\epsilon}-\log \frac{M_{H}^{2}}{\mu^{2}}+\frac{5}{6}\right)+g^{\prime 2} a_{13} \\
\Delta g=\Delta g^{\prime}=0
\end{gathered}
$$

Putting together (5.6) to (5.10) one can determine some coefficients

$$
\begin{aligned}
& a_{0}=\frac{g^{\prime 2}}{16 \pi^{2}} \frac{3}{8}\left(C_{\epsilon}-\log \frac{M_{H}^{2}}{\mu^{2}}+\frac{5}{6}\right) \\
& a_{1}+a_{13}=\frac{1}{16 \pi^{2}} \frac{1}{12}\left(C_{\epsilon}-\log \frac{M_{H}^{2}}{\mu^{2}}+\frac{5}{6}\right) \\
& a_{8}+a_{13}=0
\end{aligned}
$$

Repeating the same procedure for the three and four point Green functions one gets

$$
\begin{aligned}
& a_{2}=\frac{1}{16 \pi^{2}} \frac{1}{24}\left(C_{\epsilon}-\log \frac{M_{H}^{2}}{\mu^{2}}+\frac{17}{6}\right) \\
& a_{3}=-\frac{1}{16 \pi^{2}} \frac{1}{24}\left(C_{\epsilon}-\log \frac{M_{H}^{2}}{\mu^{2}}+\frac{17}{6}\right) \\
& a_{4}-a_{13}=-\frac{1}{16 \pi^{2}} \frac{1}{12}\left(C_{\epsilon}-\log \frac{M_{H}^{2}}{\mu^{2}}+\frac{17}{6}\right) \\
& a_{5}+a_{13}=\frac{v^{2}}{8 M_{H}^{2}}-\frac{1}{16 \pi^{2}} \frac{1}{24}\left(C_{\epsilon}-\log \frac{M_{H}^{2}}{\mu^{2}}-\frac{27 \pi}{2 \sqrt{3}}+\frac{79}{3}\right) \\
& a_{6}-a_{13}=a_{7}+a_{13}=a_{9}=a_{10}=0
\end{aligned}
$$

To determine $a_{4}$ to $a_{7}$ and $a_{10}$ we have used the Equivalence Theorem [2,20-21]. See also [22].

These values basically, but not quite, agree with those obtained in [19]. As we have discussed in this section we cannot determine with two, three and four point gauge Green functions alone the values of all coefficients $a_{i}$ in the effective chiral lagrangian. For instance $a_{1}, a_{8}$ and $a_{13}$ always appears in the combinations $a_{1}+a_{13}$ and $a_{8}+a_{13}$ while $a_{11}$ and $a_{12}$ drop from all transverse structures in the Green functions considered. Note that in the

${ }^{1}$ In the effective theory it is unnatural to demand $\hat{\Sigma}_{H}^{\prime}\left(M_{H}^{2}\right)=0$ as is usually done in the on-shell scheme, since the Higgs is integrated out. It would be better to demand a similar condition on the $\pi$ fields. However this does not affect the present calculation 
evaluation of the functional integral at the order we are working it is legitimate to use the equations of motion for the $\pi$ fields coming from the $\mathcal{O}\left(p^{2}\right)$ operators in the $\mathcal{O}\left(p^{4}\right)$ terms and, if so, $\mathcal{L}_{11}$ and $\mathcal{L}_{12}$ vanish, and $\mathcal{L}_{13}$ does not provide new independent structures, so things really are as they should.

\section{Oblique Corrections}

Although we are not able to determine with gauge Green functions alone all coefficients but only some combinations of them, these are precisely the ones that enter the physical observables. For instance, we may choose to parametrize possible departures from the Standard Model predictions in terms of the quantities $\epsilon_{1}, \epsilon_{2}$ and $\epsilon_{3}[23]$ (or $S, T, U$ [24]). Then, the contribution to these quantities from the operators $\mathcal{L}_{i}^{\prime}$ s are

$$
2 a_{0} \rightarrow \epsilon_{1} \quad-g^{2}\left(a_{8}+a_{13}\right) \rightarrow \epsilon_{2} \quad-g^{2}\left(a_{1}+a_{13}\right) \rightarrow \epsilon_{3}
$$

One way to proceed is to parametrize the 'universal' part of the radiative corrections in terms of the $\epsilon_{i}$ (basically combinations of self-energies) and use the experimental data to set constraints on them. In the SM each of the $\epsilon_{i}$ takes a well defined value and depends logarithmically on the top-quark mass. Beyond the Standard model, the Effective Chiral Lagrangian is non-renormalizable theory and some cut-off effects remain. The latter can be traced using (5.2) and (6.1) and we can form combinations that are cut-off independent, at least at the one loop level.

We prefer however to set the discussion in terms of quantities which are directly observable. LEP basically measures two quantities of relevance in the present discussion, namely the effective mixing angle $\bar{s}_{w}$, extracted from the forward-backward asymmetry $A_{F B}$ through

$$
\bar{s}_{w}^{2}=\frac{1}{4}\left(1-g_{V} / g_{A}\right) \quad A_{F B}=\frac{3}{4}\left(\frac{2 g_{V} g_{A}}{g_{V}^{2}+g_{A}^{2}}\right)^{2}
$$

and the leptonic width

$$
\Gamma_{l}=\frac{G_{F} M_{Z}^{3}}{6 \sqrt{2} \pi}\left(g_{V}^{2}+g_{A}^{2}\right)\left(1+\frac{3}{4} \frac{\alpha}{\pi}\right)
$$

$\Gamma_{l}$ is proportional to $\rho_{Z} \cdot \rho_{Z}$ parametrizes the strength of the neutral current at $s=M_{Z}^{2}$ in the improved Born approximation (at tree level $\rho_{Z}=1$ ). In this sense is the counterpart of the more familiar $\rho$ parameter (defined at $s=0$ )

$$
\mathcal{A}^{N C}\left(s=M_{Z}^{2}\right)=\rho_{Z} \sqrt{2} G_{F} M_{Z}^{2} \frac{J_{\mu} J^{\mu}}{s-M_{Z}^{2}+i \frac{s}{M_{Z}^{2}} M_{Z} \Gamma_{Z}} \quad \mathcal{A}^{N C}(s=0)=-\rho \sqrt{2} G_{F} J_{\mu} J^{\mu}
$$

The experimental data [5], assuming lepton universality and correcting for the $\tau$ mass, gives $A_{F B}=0.0170 \pm 0.0016, \Gamma_{l}=83.98 \pm 0.18 \mathrm{MeV}$, which translates into $g_{A}^{2}=0.25123 \pm$ $0.00056, g_{V}^{2}=0.00144 \pm 0.00014$ and $\bar{s}_{W}^{2}=0.23107 \pm 0.00092$. Given $m_{\text {top }}$ and $M_{H}$, the SM value is just a dot in the $\left(\bar{s}_{w}^{2}, \Gamma_{l}\right)$ plane. In any effective theory we have instead a line of points obtained by varying the (unknown) value of the cut-off. While we are unsure what value to take for it, the line itself is unambiguously predicted by one-loop 
chiral perturbation theory, independently of the regulator one uses[7]. Finding out which self-energies contribute to $g_{A}, g_{V}$ we see that, in agreement with [4], at the one loop level, we are sensitive to only one combination of coefficients in the Effective Chiral Lagrangian, namely

$$
L=-\frac{2}{9} c_{w}^{2} a_{0}+g^{2} s_{w}^{2}\left(a_{1}+a_{13}\right)+g^{2} c_{w}^{2}\left(a_{8}+a_{13}\right)
$$

From (5.11-12), in the Standard Model $L=0[4]$.

With a relatively heavy top, such as the one preliminarily reported in [25], $m_{t o p}=$ $174 \pm 10_{-12}^{+13} \mathrm{GeV}$ ) some two-loop corrections are known to be important. Sizeable contributions originate from a few genuine two-loop diagrams that yield a $m_{t o p}^{4}$ dependence[26] and from the iteration of one loop corrections through resummed propagators. The former, although not negligible, give a small contribution for our purposes. We have examined the contribution from the Effective Chiral Lagrangian to the resummed propagators checking that $a_{11}, a_{12}$ and $a_{13}$ still drop from the observables. Notice that with a two loop precision we are not entitled to appeal to the equations of motion derived from the $\mathcal{O}\left(p^{2}\right)$ terms. The set of points obtained by varying $\log M_{H}$ (or $\Lambda$ in the effective theory) is no longer a straight line, but deviations are really not perceptible.

The results are shown in fig. 2. We have plotted in addition of $L=0$ (the value in the Minimal Standard Model) lines for the values $L=-e^{2} / 12 \pi^{2}$ and $L=-e^{2} / 6 \pi^{2}$. These correspond to theoretical estimates[27] for $L$ in one-generation technicolor models with $N_{T C}=2,4$, respectively. The same estimates in QCD would give values which are between $30 \%$ and $40 \%$ below the experimental results, so we regard those as lower bounds. (A fact that can be rigorously established in the large $N_{T C}$ limit.) These lines do not agree in slope with the one presented in [28].

Unless one is willing to make somewhat uncertain extrapolations from QCD, the model with $N_{T C}=4$ and one full generation of technifermions is not quite excluded at the $99 \%$ confidence level, a conclusion that somehow runs contrary to a widespread belief. A model with $N_{T C}=2$ falls within the $68 \%$ c.l. boundary. Even allowing for somewhat larger values for $L$ it is hard to convincingly exclude this model at this point.

In conclusion, we have revised thoroughly the procedure by means of which one determines the coefficients in an Effective Chiral Lagrangian that reproduce the Standard Model in the large $M_{H}$ limit. We have pointed out a number of subtleties regarding gauge invariance, commutation of limits and the precise formulation of the matching conditions. At the end, we can determine all experimentally relevant coefficients (but not all coefficients). We have included the leading two loop corrections to take a fresh look at the issue whether LEP data really excludes technicolor models or not with the current level of precision and the preliminary determination of $m_{t o p}$. 


\section{Acknowledgements}

We thank M.J.Herrero and H.Leutwyler for discussions that have triggered different parts of this work. The thank very specially M.Martinez who has helped us in the analysis of the experimental data. We acknowledge the financial support from CICYT grant AEN930695 and CEE grant CHRX CT93 0343. J.M. acknowledges a fellowship from Ministerio de Educacion y Ciencia. D.E. would like to thank C.Garcia-Canal and the Theory Group at Universidad de La Plata for the hospitality extended to him.

\section{References}

[1] T.Appelquist and C.Bernard, Phys. Rev. D22 (1980) 200; A.Longhitano, Phys. Rev. D22 (1980) 1166; A. Longhitano, Nucl. Phys. B188 (1981) 118

[2] M. Chanowitz and M.K. Gaillard, Nucl. Phys. B261 (1985) 379; G.J. Gounaris, R. Kogerler and H. Neufeld, Phys. Rev. D34 (1986) 3257; M. Chanowitz, M. Golden and H.Georgi, Phys.Rev. D36 (1987) 1490; O.Cheyette and M.K.Gaillard, Phys. Lett B197 (1987) 205; Y.P.Yao and C.P.Yuan, Phys. Rev. D38 (1988) 2237; H.Veltman, Phys. Rev. D41 (1990) 2294

[3] R. Renken and M.Peskin, Nucl. Phys. B211 (1983) 93; T.Appelquist, T. Takeuchi, M. Einhorn and L.C.R. Wijewardhana, Phys. Lett. B 232 (1989) 211; A.Dobado and M.J.Herrero, Phys.Lett. B228 (1989) 495; J.F.Donoghue and C.Ramirez, Phys. Lett. B234 (1990) 361; B.Holdom and J.Terning, Phys. Lett. B247 (1990) 88; A.Dobado and M.J.Herrero and J.Terron, Z.Phys. C50 (1991) 205, 465; S.Dawson and G.Valencia, Nucl.Phys. B352 (1991) 27; M.Golden and L.Randall, Nucl. Phys. B361 (1991) 3; T. Appelquist and G. Triantaphyllou, Phys. Lett. B278 (1992) 345; J.Bagger, S.Dawson and G.Valencia, Fermilab-Pub-92/75-T,1992; T. Appelquist and G-H. Wu, Phys.Rev.D 48 (1993) 3235

[4] A.Dobado, D.Espriu and M.J. Herrero, Phys. Lett. B255 (1991) 405

[5] Internal Note LEPEWWG/94-01 ALEPH 94-74 PHYSIC 94-63,DELPHI 94-33 PHYS 364 L3 Note 1599, OPAL Technical Note TN235, May (1994)

[6] R.Haag, Phys. Rev. 112 (1958) 669;S.Coleman, J.Wess and B.Zumino, Phys.Rev.177 (1969) 2239;C.G.Callan, S Coleman, J.Wess and B.Zumino, Phys.Rev. 177 (1969) 2247

[7] D. Espriu and J.Matias, Nucl. Phys. B 418 (1994) 494

[8] J.Honerkamp and K.Meetz, Phys. Rev. D3 (1971) 1996; G.Ecker and J.Honerkamp, Nucl. Phys. B 35(1971) 481; 52(1973) 211; 62 (1973) 509; T. Appelquist and C.Bernard, Phys. Rev D 23 (1981) 425

[9] G. Burgers and W. Hollik, in Polarization at LEP, CERN Yellow Report, ed. G. Alexander et al. (CERN, Geneva, 1988); M.Consoli and W.Hollik, in Z Physics at LEP1, CERN Yellow Report, ed. G.Altarelli et al. (CERN, Geneva, 1989) G.Burgers and F.Jegerlehner, ibid

[10] M.Bohm,H.Spiesberger and W.Hollik, Fortschr. Phys. 34 (1986) 687

[11] W.J.Marciano and A.Sirlin, Phys.Rev. D22 (1980) 2695, J.Fleischer and F.Jegerlehner Nucl.Phys. B 228 (1983) 1

[12] K.I.Aoki, Z.Hioki,R.Kawabe,M.Konuma and T.Muta, Suppl. of the Progress of Theoretical Physics 73 (1982) 1 
[13] G. Degrassi and A.Sirlin, Nucl.Phys. B352 (1991) 342; P.Gambino and Phys.Rev. D49 (1994) 1160

[14] B.Ovrut and H.Schnitzer,Phys. Rev. D21 (1980) 3369; Phys. Rev. D22 (1980) 2518; Phys. Rev. D24 (1981) 1695 and references therein

[15] D. Espriu and M.J. Herrero, Nucl. Phys. B 373 (1992) 117

[16] M.J.Herrero and E.R. Morales, Phys.Lett. B296 (1992) 397

[17] H.Georgi, in Proc. of the Workshop on Effective Field Theories of the S.M., Dobogoko, Hungary, August 1991 (ed. U-G. Meissner, World Scientific); H.Georgi, L.Kaplan and D.Morin , Phys.Rev. D49 (1994) 2457

[18] F.Feruglio in Lectures at the $2^{\text {nd }}$ NATO Seminar, Parma, Univ. di Padova (1992) DFPD92-TH- $/ 50$

[19] M.J.Herrero and E.R.Morales, Nucl.Phys. B418 (1994) 431

[20] A. Dobado and J.R. Pelaez Preprint SU-ITP-93-33 (to be published in NPB) and Phys.Lett. B329 (1994) 469; D. Espriu and J. Matias, in preparation

[21] M.J.G. Veltman and F.J.Yndurain, Nucl.Phys. B325 (1989) 1; S. Dawson and S. Willenbrock, Phys. Rev. Lett. 62 (1989) 1232

[22] M.J.Herrero and E.R.Morales, Preprint Universidad Autonoma de Madrid, (1994) FTUAM 94/11 and FTUAM 94/12

[23] G.Altarelli and R.Barbieri, Phys. Lett. B253 (1991) 161; G.Altarelli and R.Barbieri and S.Jadach, Nucl.Phys. B369 (1992) 3

[24] M.Peskin and T.Takeuchi, Phys. Rev. Lett. 65 (1990) 964

[25] F.Abe et al. The CDF Collaboration FERMILAB Pub-94/097-E CDF

[26] R. Barbieri et al., Phys. Lett. B288 (1992) 95; J. Fleischer, O.V. Tarasov and F.Jegerlehner, Phys. Lett. B319 (1993) 249

[27] D.Espriu, E. de Rafael and J.Taron, Nucl.Phys. B345 (1990) 22

[28] D.Buskulic et al.,The ALEPH Collaboration, Z.Phys. C60 (1993) 71 


\section{Figure Captions}

Fig. 1.- a) One-Particle-Irreducible diagrams that enter the $W^{+} W^{-} Z$ (or $A$ ) vertex. b) Reducible diagrams that restore the equality of the connected gauge Green functions, as discussed in the text.

Fig. 2.- Plot of $\Gamma_{l}$, the leptonic width, versus the effective mixing angle $\bar{s}_{w}^{2}$ for $m_{\text {top }}=174 \pm 10$ Gev and $100 \mathrm{GeV} \geq M_{H} \leq 1500 \mathrm{Gev}$ in the Minimal Standard Model (solid line). The leading two-loop corrections have been included. The dashed line (A) corresponds to the same quantity (again including the leading two-loop corrections) calculated in an Effective Lagrangian for $m_{t o p}=174$. The agreement is exact when $M_{H} \rightarrow \infty$. We then modify the coefficients of the Effective Chiral Lagrangian to include QCD-like models for the symmetry breaking sector with $N_{T C} \times N_{D}=8(\mathrm{~B})$ and $16(\mathrm{C})$. The values chosen correspond to theoretical calculations that are really lower bounds in vector-like models. The elipsis corresponds to the experimental data with $68 \%$ and 99\% C.L. The one-loop SM results are also shown (dotted line). 
This figure "fig1-1.png" is available in "png" format from: http://arxiv.org/ps/hep-ph/9407292v2 
This figure "fig2-1.png" is available in "png" format from: http://arxiv.org/ps/hep-ph/9407292v2 